\title{
First direct comparative test of single crystal rhodium and molybdenum mirrors for ITER diagnostics
}

\author{
A. Litnovsky ${ }^{\mathrm{a}}$, Yu. Krasikova ${ }^{\mathrm{a}}$, M. Rasinski ${ }^{\mathrm{a}}$, A. Kreter ${ }^{\mathrm{a}}$, Ch. Linsmeier ${ }^{\mathrm{a}}$, Ph. Mertens ${ }^{\mathrm{a}}$, B. Unterberg ${ }^{\mathrm{a}}$, \\ U. Breuer $^{\mathrm{b}}$ and T. Wegener ${ }^{\mathrm{a}}$
}

${ }^{a}$ Forschungszentrum Jülich GmbH, Institut für Energie- und Klimaforschung - Plasmaphysik, Partner of the Trilateral
Euregio Cluster (TEC), 52425 Jülich, Germany;
${ }^{b}$ Forschungszentrum Jülich GmbH, Central Institute for Engineering, Electronics and Analytics ZEA-3, 52425 Jülich, Germany.

\begin{abstract}
All optical and laser diagnostics in ITER will use mirrors to observe the plasma radiation. In the ITER environment mirrors may become contaminated with plasma impurities hampering the performance of corresponding diagnostics. An in-situ mirror cleaning is proposed, which relies on ion sputtering of the contaminants and the affected mirror material. Previous research demonstrated the advantages of single crystal (SC) molybdenum (Mo) under sputtering conditions over polycrystalline concepts. Recently, the first single crystal rhodium (Rh) mirrors became available and tests have been started at the Forschungszentrum Jülich.

In a direct test SC Rh and SC Mo mirrors were exposed under identical conditions in steady-state helium plasmas in the linear plasma device PSI 2. The energy of impinging ions was $~ 100 \mathrm{eV}$ matching conditions expected in the in situ cleaning system in ITER. During exposure molybdenum mirrors lost 420-500 nm due to sputtering. Rhodium mirrors lost about $1 \mu \mathrm{m}$. Exposure corresponded to 50 - 100 cleaning cycles in ITER. Nevertheless, rhodium mirrors have preserved their specular reflectivity, showing the maximum degradation of less than $7 \%$ at $250 \mathrm{~nm}$. Molybdenum mirrors demonstrated a moderate decrease of specular reflectivity of $12 \%-25 \%$. Results open new perspectives for the use of single crystals in ITER diagnostics.
\end{abstract}

Keywords: ITER diagnostic mirrors, mirror cleaning, single-crystal rhodium, single-crystal molybdenum, sputterresistant mirrors, recovery of reflectivity,

\section{Introduction and motivation}

Plasma - viewing mirrors are crucially important elements of optical and laser-based diagnostics of ITER. In the harsh particle, radiation and neutron environment of ITER, the use of conventional lens-based optics is impossible due to the rapid degradation of their optical performance. Therefore, the plasma radiation will be observed by mirror-based systems which transmit the detected light to detectors, located far from plasma. There will be about 26 optical diagnostics in ITER, where mirrors will be used. Those diagnostics are supposed to measure the plasma and technical parameters which are vital for the operation of ITER, including concentrations and temperatures both of the main plasma ions and impurities, temperature of plasmafacing components in the main chamber and in the divertor. Some of these systems are involved in machine safety and basic operation [1, 2]. There will be around 80 first mirrors to be installed both in the main chamber and in the divertor. Optical reflectivity of the mirror is the crucial parameter, immediately affecting the overall performance of the corresponding diagnostic systems. The extensive research made on several tokamaks and on lab-scale plasma systems allowed to detect to main risks for the mirror performance and to plan measures for their mitigation [3-6].

The deposition of plasma impurities causes the strongest effect on the mirror reflectivity. In order to counteract the deposition, a set of specific measures is planned. One of these measures foresees the use of an in situ mirror cleaning system to remove the deposits from the mirror surface and thus to recover the reflectivity. Presently, cleaning by plasma sputtering is accepted as the most advanced and realizable system [7]. However, the use of a plasma cleaning system imposes additional criteria, in particular on mirror material and on the mirror design. In order to conform both to high precision measurements and to the recovery of the reflectivity, the mirror material must have high reflectivity and excellent sputtering resistance.

Presently, the single crystal molybdenum (SC Mo) is widely accepted as the first mirror for the majority of diagnostics in ITER. SC Mo demonstrated superior performance both in the lab and tokamak experiments [8, 9]. As a drawback, molybdenum possesses rather moderate reflectivity, especially in the VIS- UV range 250-800 nm [10]. There is another highly reflecting material of interest - rhodium (Rh). Rhodium possesses a much higher reflectivity than that of molybdenum. Rh is also an expensive element. The investigations were therefore limited to the thin polycrystalline $\mathrm{Rh}$ films on the substrate material (e.g. tungsten or molybdenum). Studies show the limited potential of polycrystalline mirrors. The surface roughness of the polycrystalline mirror samples increased severely in the course of sputtering leading to a dramatic drop of the specular reflectivity $[8,9,11]$ and thus leaving no chance for a signal detection. However, since recently the single crystal (SC) Rh-samples became available and experimental investigations became feasible. In this 
paper the results of the first direct comparative test of single crystal molybdenum and rhodium mirrors are presented. The aim of this test was to study the resistance to sputtering and the effect of sputtering on optical properties of the aforementioned two candidate mirror materials.

\section{Experimental}

\subsection{Mirror samples and plasma exposure}

For the exposure, two SC Mo mirrors and two SC $\mathrm{Rh}$ mirrors were produced. All the mirrors had the orientation [100] and all were polished using the same diamond turning procedure to the final surface roughness $\mathrm{R}_{\mathrm{a}} \sim 2-4 \mathrm{~nm}$. The mirrors had a thickness of $4 \mathrm{~mm}$, and a diameter of $22 \mathrm{~mm}$. The round polished surface of the mirror had a diameter of $18 \mathrm{~mm}$. Series of measurements were made on the mirrors before and after exposure. These measurements comprised studies of the total and diffuse reflectivity and investigations of surface morphology and elemental composition. The surface roughness $R_{a}$ was evaluated using the stylus profiler Dektak 6M from Bruker in the MirrorLab [12]. Scan locations are shown in Fig.1a. Each scan of surface roughness consisted of five measurements, the final result was averaged. Time-of-flight Secondary Ion MassSpectrometry (ToF SIMS) investigations were made in the middle of each sample using a ToF-SIMS IV (IONTOF GmbH, Münster, Germany) facility on locations shown in Fig 1a. SIMS measurements provided a depth profiling of the elemental composition of all samples. The investigations were made with $2 \mathrm{keV} \mathrm{Cs}^{+}$ sputter ions. Scanning electron microscopy (SEM) surface surveys were made on each sample on the area shown in Fig. 1a using the Carl Zeiss CrossBeam XB 540 microscope equipped with the focused ion beam (FIB). Cross-section viewing by FIB was performed in the central area of each sample shown with the smallest square in Fig.1a. Special markers were made with an ion beam on the side surface of each FIB crater to directly measure the material sputtered during plasma exposure.

After pre-characterization, the mirrors were installed pair by pair into the mirror holder and mounted in the sample transfer system of the PSI 2 linear plasma device [13]. The samples were exposed to the steadystate helium plasmas. The mirror holder was biased to $\mathrm{U}=-130 \mathrm{~V}$, the corresponding energy of impinging ions was $\sim 100 \mathrm{eV}$ after the correction made on repelling plasma potential. The energy of impinging helium ions was intentionally chosen to match the energy expected in in situ cleaning plasma system for ITER diagnostics. Plasma parameters were studied with the moveable Langmuir probe system. The electron temperature during the exposure was $7-8 \mathrm{eV}$, plasma flux $2 \times 10^{18} \mathrm{He} / \mathrm{cm}^{2}$ per second. The total duration of the exposure was 16 minutes resulting in the accumulated averaged fluence of He ions of $1.9 \times 10^{21} \mathrm{He} / \mathrm{cm}^{2}$. The temperature of mirrors during exposure was monitored with a FLIR SC7500 infrared camera and was detected to be in the range of $240^{\circ} \mathrm{C}-280^{\circ} \mathrm{C}$. A photo of mirrors during plasma exposure is presented in Fig.1b.

\subsection{Results of exposure}

After exposure the mirrors were cooled down in the plasma facility under vacuum. Then the mirror samples were dismounted and underwent the series of post - exposure investigations. For the beginning, the mass change of the samples was studied. The corresponding data is provided in Table 1 . The calculated mass loss was used to estimate the amount of sputtered material. The molybdenum mirrors were measured to lose $420-480 \mathrm{~nm}$ of material during the exposure, whereas rhodium mirrors lost $1 \mu \mathrm{m}$.

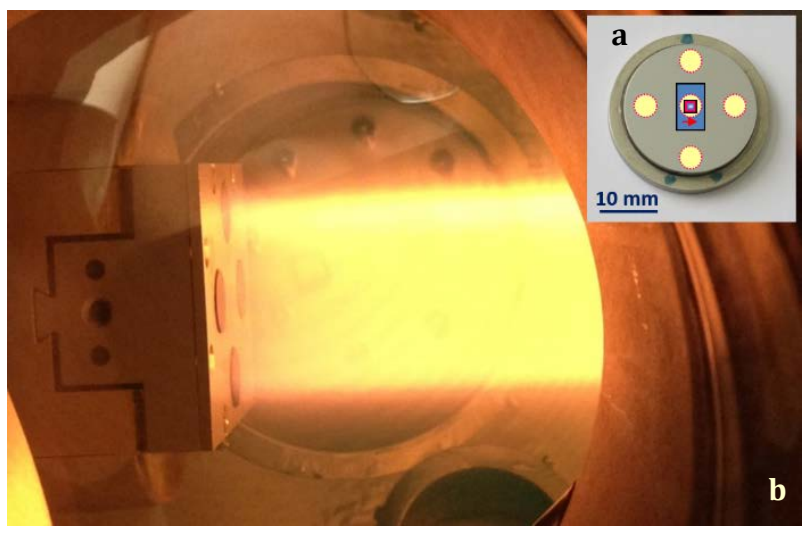

Fig.1. a) Mirror arrangement and measurement locations: round spots represent the locations of the total reflectivity $\mathbf{R}_{\text {tot }}$ measurements, the large rectangular block outlines the location of the diffuse reflectivity measurements, the largest square represents the location of the SIMS measurement, whereas the smaller square shows the location of the SEM-FIB investigations, location of surface roughness scans is shown with an arrow; b) SC Rh and SC Mo mirrors during exposure in PSI 2.

The measured mass loss was used to investigate the sputtering coefficient experimentally. The experimental results were compared with the literature data. Perfect agreement of experimental and literature data [14] on sputtering of molybdenum was attained. The sputtering coefficient of rhodium also matches very well the literature [14].

During the pre-characterization, 5-10 $\mu$ m-wide craters were milled with FIB in the middle of each mirror. In addition, on the side of each crater, fine marker lines were milled every $1 \mu \mathrm{m}$ in the depth of each mirror. The craters with markers can be seen in Figure 2. After exposure the craters were detected and inspected using SEM cross-section viewing. The missing markers yielded directly an amount of sputtered material.

According to analyses of FIB craters, the molybdenum lost about $500 \mathrm{~nm}$ due to sputtering, whereas single crystal Rh lost about $1000 \mathrm{~nm}$. Such an independent and direct measurement of eroded material underlines the perfect agreement between estimates of sputtering coefficient made using the integral evaluation from the weight loss reported earlier. 
Table 1. Results of sputtering of single crystal mirrors

\begin{tabular}{|c|c|c|c|c|c|c|}
\hline Mirror & $\begin{array}{c}\text { Mass loss, } \\
\text { mg }\end{array}$ & $\begin{array}{c}\text { Sputtered } \\
\text { material, nm }\end{array}$ & $\begin{array}{c}\text { Sputtering } \\
\text { coefficient, } \\
\text { at/ion. }\end{array}$ & $\begin{array}{l}\text { Literature } \\
\text { sputtering } \\
\text { data }\end{array}$ & $\begin{array}{l}\text { Roughness } \\
\text { before } \\
\text { exposure, nm }\end{array}$ & $\begin{array}{c}\text { Roughness } \\
\text { after exposure } \\
\mathrm{nm}\end{array}$ \\
\hline SC Mo PSI 1 & 1.2 & $480 \mathrm{~nm}$ & $1.6^{*} 10^{-3}$ & \multirow{2}{*}{$1.5^{*} 10^{-3}$} & 2,37 & 3,87 \\
\hline SC Mo PSI 2 & 1.05 & $420 \mathrm{~nm}$ & $1.4^{*} 10^{-3}$ & & 2,30 & 4,24 \\
\hline SC Rh PSI 3 & 2.97 & $984 \mathrm{~nm}$ & $3.7^{*} 10^{-3}$ & \multirow{2}{*}{$5.0^{*} 10^{-3}$} & 1,22 & 1,39 \\
\hline SC Rh PSI 4 & 3.04 & $1008 \mathrm{~nm}$ & $4.0^{*} 10^{-3}$ & & 2,26 & 2,10 \\
\hline
\end{tabular}

Series of SEM surveys were performed on the surface of the mirrors before and after exposure. The respective SEM photos are provided on Figure 3. It can be readily seen that the surface roughness after exposure contains enhanced structure - which is typical for exposure to high-energy helium ions of $100 \mathrm{eV}$. There are crater-like structures at the surface of the mirror. The size of such crater is in the order of a micrometer. The observed enhancement of surface structure would lead to the increased surface roughness as confirmed by the dedicated investigations made with stylus profiler, presented in Table 1.

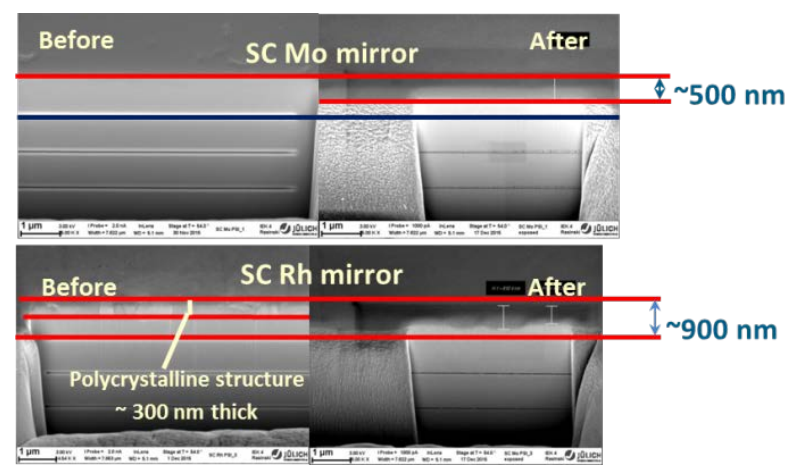

Fig 2. Cross-section viewing of FIB craters before and after exposure. The material lost via sputtering is shown with scale and dimensions.

The enhanced structure observed after exposure and the corresponding increase of surface roughness would have led to an increase of the diffuse reflectivity.

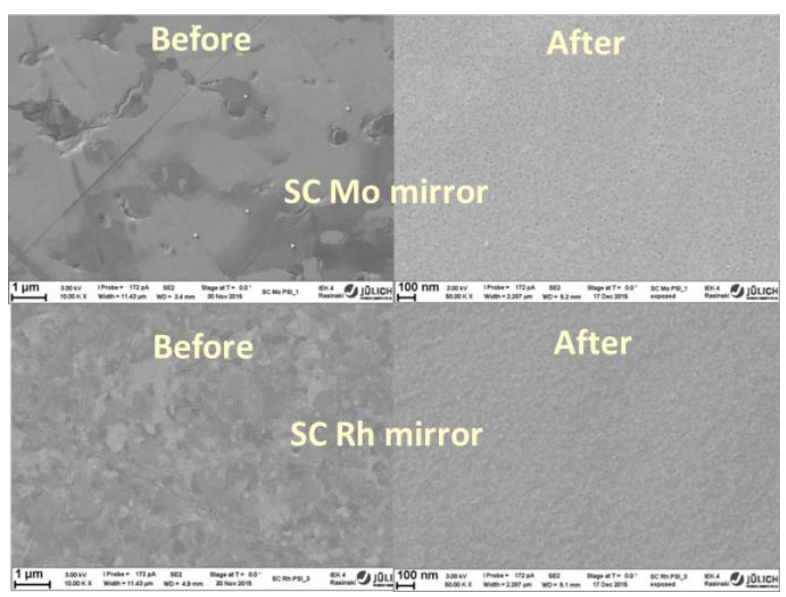

Fig. 3. Surface morphology of SC mirrors before and after exposure reflectivity on the wavelength are provided in Figure 4 for both SC Mo and SC Rh mirrors. As it can be seen, in case of SC Mo, the significant enhancement of the diffuse reflectivity was indeed observed after the exposure. The increase of $\mathrm{R}_{\text {diff }}$ has reached $7 \%$ at 250 $\mathrm{nm}$. At the same time, no increase of diffuse reflectivity was observed for both SC Rh mirrors, despite of the 1 $\mu \mathrm{m}$ of removed material. This is a remarkable result. The dependencies of specular reflectivity on the wavelength are shown in Figure 5 for both SC Mo and SC Rh samples. The decrease of reflectivity of the SC Mo mirrors is the most pronounced in the UV range reaching $\sim 22 \%$ at the $250 \mathrm{~nm}$. The maximum decrease of specular reflectivity however, did not exceed $10 \%$.

SC Rh revealed only insignificant degradation of their reflectivity, reaching $7 \%$ at its maximum at 250 $\mathrm{nm}$. In the rest of the wavelength region of investigation, the decrease of specular (directed) reflectivity over the entire wavelength range of interest does not exceed 2-3 percent, which is an excellent result.

\section{Summary}

The single crystal rhodium mirrors have just become available. The first direct comparative test with single crystal Rh and Mo mirrors under identical conditions was made. Mirrors were biased and exposed to helium stationary plasma, the energy of plasma ions were tuned up to the range expected in in situ mirror cleaning system in ITER diagnostics.
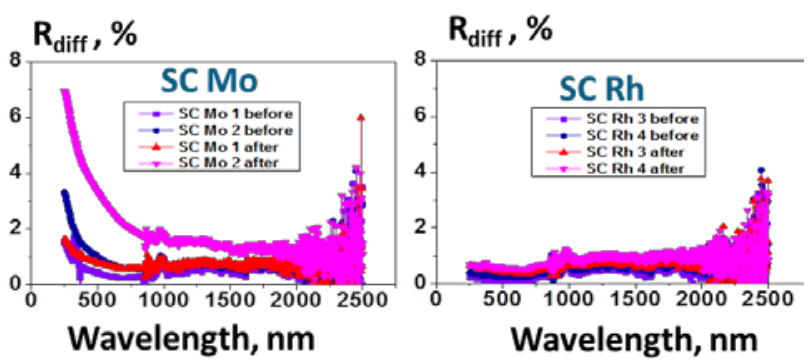

Fig.4. The dependence of the diffuse reflectivity $\mathrm{R}_{\text {diff }}$ on the wavelength for SC Mo and SC Rh mirrors before and after plasma exposure.

In the course of exposure, the single crystal Mo mirror has lost about $500 \mathrm{~nm}$ of material. For the SC Rh 
the loss of material corresponded to about a $1 \mu \mathrm{m}$ removed by physical sputtering. The material loss estimate corresponds very well to the literature data. Moreover, the estimate of removed material due to sputtering: $\sim 500 \mathrm{~nm}$ for SC Mo and $1000 \mathrm{~nm}$ for SC $\mathrm{Rh}$ agrees perfectly with the results of the direct measurements of sputtered material.

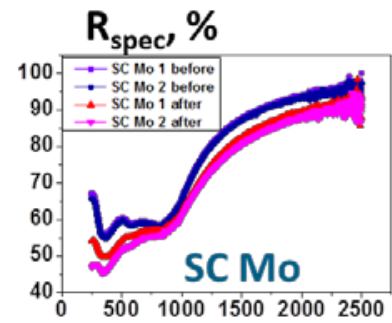

Wavelength, $\mathrm{nm}$

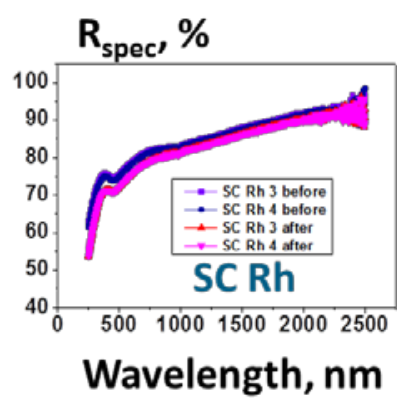

Fig.5. The dependence of the specular reflectivity $\mathrm{R}_{\text {spec }}$ on the wavelength for SC Mo and SC Rh mirrors before and after plasma exposure.

This amount of removed material when normalized on the material supposed to be lost in the course of mirror cleaning in ITER corresponds to about 50-100 cleaning cycles. Under these conditions the single crystal Mo mirrors demonstrated moderate degradation of their specular reflectivity especially in the UV range. The decrease of reflectivity resulted in its maximum to $22 \%$ at $250 \mathrm{~nm}$, whereas at maximum a $\mathbf{1 0 \%}$ decrease was detected in the rest of the studied wavelength region.

SC Rh mirror exposed under identical conditions featured very moderate decrease of specular reflectivity, not exceeding $7 \%$ in the UV. Having summarized the results of this first test, the conclusion can be made that under conditions relevant to the in-situ mirror cleaning system for ITER, the newly available single crystal Rh mirrors outperformed the single crystal Mo mirror samples.

\section{Outlook}

Future studies will be focused on better understanding of the operational limits for both single crystal systems. The dedicated investigations will be made for different crystal orientations in order to find the orientation featuring the least sputtering and morphology change during the mirror cleaning. New sputtering tests will be made at higher fluence of helium ions in order to attain the limit of application of the single crystal $\mathrm{Rh}$ mirrors. Subsequently, both single crystal mirror materials will be tested under steady-state sputtering argon plasmas - in order to address the cleaning scenario expected for removal of tungsten-containing deposits. In future experiments, special care has to be taken for the initial preparation of the mirrors in order to avoid the polycrystalline layers produced during polishing.

\section{Acknowledgmeents}

The authors would like to thank S. Kraus and M. Vogel for the technical assistance and help in preparation and conduction of the experiment.

\section{References:}

[1]. E. Costley "Requirements and issues in diagnostics for next step burning plasms experiments", Int. Conf. on adv. diagnostics for magnetic and inertial fusion, Varenna, Italy, September 2001;

[2]. A. Litnovsky, V.S. Voitsenya, D. Thomas et al., "Mirrors for ITER diagnostics: new R\&D developments, assessment of the mirror lifetime and impact of the mirror failure on ITER performance”, proceedings of the 23rd IAEA Fusion Energy Conference Proceedings 1016 October 2010, Daejon, Republic of Korea, ITR/P1-05;

[3]. M.Lipa, B. Schunke, Ch. Gil et al., Fus. Eng. and Design 81 (2006) 221;

[4]. D. Ivanova, M.Rubel, A. Widdowson et al, Phys. Scr. T159 (2014) 014011;

[5]. V.S. Voitsenya and A. Litnovsky, Plasma Dev. and Operations, Vol. 17, No. 4, December 2009, 309;

[6]. A. Litnovsky, P. Wienhold, V. Philipps et al, J. Nucl. Materials, 363-365 (2007) 1395

[7]. F. Leipold, R. Reichle, V. Vorpahl et al., Rev. Sci. Instr. 87 (2016) 11D439

[8]. V.S. Voitsenya, A.E. Costley, V. Bandourko et al., Rev. Sci. Instrum. 72, (2001) 475;

[9]. A.Litnovsky, G. De Temmerman, K. Yu. Vukolov et al., Fus. Eng. and Design 82 (2007) 123;

[10]. Handbook of Optical Constants of Solid, $2^{\text {nd }}$ Edition, edited by E. D. Palik, Academic press, New York, 1985.

[11]. M. Matveeva, A. Litnovsky, L. Marot et al., "Material choice for first ITER mirrors under erosion conditions", Proceedings of the $37^{\text {th }}$ EPS Conference on Plasma Physics, Dublin, Ireland, 21 - 25 June, 2010, P2.105

[12]. MirrorLab Website: https://tec.ipp.kfajuelich.de/mirrorlab/, Access details: mirrorlab@fzjuelich.de;

[13]. A.Kreter, C. Brandt, A. Huber et al., Fusion Sci. Technol. 68(2015)8;

[14]. Y. Yamamura and H. Tawara Atomic data and nuclear data tables, 149-253 (1996) 